\title{
Efectividad del gel de clorhexidina y pasta de hidróxido de calcio con clorhexidina como medicamento intraconducto en la eliminación del Enterococcus faecalis
}

\section{Effectiveness of chlorhexidine gel and calcium hydroxide paste with chlorhexidine as intracanal medicaments in Enterococcus faecalis elimination}

\section{Artículo de Revisión}

\author{
Andrea Carolina Martínez Guzhñay ${ }^{1, a}$, María Emilia \\ Guerrero Coello ${ }^{1, b}$ \\ ${ }^{1}$ Universidad Católica de Cuenca, Facultad de Odontología, \\ Cuenca, Ecuador \\ a Bachiller en Odontología. \\ ${ }^{\mathrm{b}}$ Especialista en Endodoncia.
}

\section{Correspondencia:}

Andrea Carolina Martínez Guzhñay: acaromartinez1998@ gmail.com

Universidad Católica de Cuenca. Av de las Américas y

Humboldt, Cuenca, Ecuador.

ORCID: 0000-0003-1316-9502

\section{Coautores:}

María Emilia Guerrero Coello: mguerroc@ucacue.edu.ec ORCID: 0000-0001-5640-9575

Editor:

Donald Ramos Perfecto

Universidad Nacional Mayor de San Marcos, Perú.

Conflicto de intereses: los autores declaran no tener conflictos de interés.

Fuente de financiamiento: autofinanciado.

\section{Recibido: 28/03/21}

Aceptado: 11/05/21

Publicado: 05/10/21

El objetivo de este estudio es conocer la efectividad del gel de clorhexidina y pasta de hidróxido de calcio con clorhexidina como medicamento intracanal en la eliminación del Enterococcus faecalis (E. faecalis). Se realizó una revisión bibliográfica mediante una búsqueda exhaustiva en diferentes bases de datos como: PubMed, ScienceDirect, SciELO, Springer, Google Scholar. Se seleccionaron mediante los criterios de inclusión y exclusión 37 artículos publicados en el periodo 2003-2020, los cuales fueron enfocados especialmente en $E$. faecalis y medicación intracanal. Teniendo como resultados que la clorhexidina en mayor porcentaje resultó ser el medicamento intracanal más eficaz en un tiempo promedio de 7 días, seguida de la combinación de hidróxido de calcio más clorhexidina que actuó de igual manera eliminando el $E$. faecalis de los canales radiculares, mientras que el hidróxido de calcio no presentó actividad antimicrobiana frente al E. faecalis.

Palabras clave: Hidróxido de calcio; Clorhexidina; Conducto radicular; Tratamiento, Enterococcus faecalis. (fuente: DeCS BIREME).

\begin{abstract}
The aim of this study was to know the effectiveness of chlorhexidine gel and calcium hydroxide paste with chlorhexidine as intracanal medicaments in Enterococcus faecalis (E. faecalis) elimination. A bibliographic review was done through an exhaustive search in different databases such as: PubMed, ScienceDirect, Scielo, Springer, Google Scholar. Using inclusion and exclusion criteria, 37 articles published in the period 2003-2020 were selected, which were especially focused on E. faecalis and intracanal medication. The analysis showed that chlorhexidine in a higher percentage was the most effective intracanal medication in an average time of 7 days, followed by the combination of calcium hydroxide plus chlorhexidine that acted in the same way eliminating E. faecalis from the root canals, while calcium hydroxide did not show any antimicrobial activity against E. faecalis.
\end{abstract}

Keywords: Calcium hydroxide; Chlorhexidine; Root canal; Treatment, Enterococcus faecalis (source: MeSH NLM).

(C) Los autores. Este artículo es publicado por la revista Odontología Sanmarquina de la Facultad de Odontología, Universidad Nacional Mayor de San Marcos. Este es un artículo de acceso abierto, distribuido bajo los términos de la licencia Creative Commons Atribución 4.0 Internacional (CC BY 4.0) [https://creativecommons.org/ licenses/by/4.0/deed.es] que permite el uso, distribución y reproducción en cualquier medio, siempre que la obra original sea debidamente citada de su fuente original. 


\section{Introducción}

Uno de los motivos de fracaso endodónticos es la eliminación parcial del tejido pulpar y la permanencia de microorganismos en los canales radiculares. Los microorganismos persisten, incluso después de realizar la preparación químico mecánica e invaden los canales radiculares de raíces obturadas mediante una filtración coronal. La bacteria identificada con frecuencia en tratamientos endodónticos aplicados por primera vez, o que presentan recidiva de infección es el Enterococcus. faecalis (E. faecalis) ${ }^{1}$.

E. faecalis es una bacteria Gram positiva, anaerobia facultativa, inmóvil no esporulado y habitante normal del tracto gastrointestinal del ser humano. Bacteria que es identificada como responsable de infecciones periapicales persistentes, debido a ciertas cualidades identificadas como: gran capacidad de desarrollo y supervivencia en ambientes tóxicos con temperaturas elevadas, supervivencia en conductos radiculares de dientes tratados endodónticamente ${ }^{1,2}$.

La limpieza y el modelado del conducto radicular constituye un procedimiento que reduce la población bacteriana; además la medicación intraconducto es considerada como una alternativa para la eliminación de $E$. faecalis. La Medicación Intraconducto consiste en colocar un agente antimicrobiano en el canal radicular entre citas, cuyo propósito radica en destruir los microorganismos restantes y prevenir la reinfección ${ }^{2}$. Por lo tanto, se utiliza para matar bacterias, reducir la inflamación y el dolor, también ayuda a eliminar el exudado apical, controlar la reabsorción de la raíz y prevenir la contaminación en cada uno de los procedimientos. Cuando no se usan medicamentos intracanales, el número de bacterias aumenta rápidamente ${ }^{3}$.

Numerosas sustancias se disponen para ser usadas como medicación intraconducto; el Hidróxido de Calcio (HC) y la Clorhexidina juegan un papel importante en endodoncia por la capacidad de impulsar la formación de tejido duro, disolver tejidos, además de imposibilitar la reinfección del canal radicular ${ }^{3}$.

El HC es el medicamento intracanal frecuentemente utilizado en tratamientos endodónticos, la propiedad antimicrobiana se atribuye a la liberación de iones hidroxilo y proporciona un entorno altamente alcalino con valores de $\mathrm{pH}$ aproximadamente 12,5. En este sentido, los microorganismos que se encuentran en los canales radiculares infectados no sobreviven en el medio alcalino, sin embargo, el HC no es totalmente eficaz contra todas las bacterias que se encuentran en el canal radicular ${ }^{4}$.

El gluconato de clorhexidina (CHX) se utiliza en tratamientos endodónticos como medicamento intracanal, tomando en cuenta la biocompatibilidad y amplia actividad antimicrobiana, la CHX es un agente eficaz contra las bacterias Gram positivas, Gram negativas y contra los microorganismos resistentes al $\mathrm{HC}^{4}$. A bajas concentraciones la CHX es bacteriostática; sin embargo, cuando se presenta en altas concentraciones tiene un efecto bactericida ${ }^{5}$.
Varios estudios buscan comparar el efecto antibacteriano del HC solo, o en combinación con la CHX. Los resultados de algunos estudios demuestran mayor efecto antibacteriano cuando se agrega $\mathrm{CHX}$ al $\mathrm{HC}$, mientras que otros estudios evidencian ningún beneficio al incorporar $\mathrm{CHX}^{5}$. $\mathrm{Al}$ respecto, la presente investigación tuvo como objetivo conocer la efectividad del gel de CHX y pasta de HC con CHX como medicamento intraconducto en la eliminación del E. faecalis.

\section{Metodología}

Se realizó una revisión de la literatura dividida en las siguientes etapas: (i) Estrategia de búsqueda, (ii) Periodo de búsqueda, (iii) Selección de artículos relevantes (criterios de inclusión y exclusión), (iiii) Extracción de datos.

La búsqueda de artículos relevantes se basa en frases conformadas por las palabras clave (en inglés): "calcium hydroxide, chlorhexidine, intracanal medication, Enterococcus Faecalis. La cadena de búsqueda definida fue: "Clorhexidine AND calcium hydroxide AND intracanal medication AND Enterococcus faecalis". Los documentos relacionados con el área de investigación se seleccionaron de revistas, revisiones sistemáticas, investigaciones propias u originales. Se utilizaron las siguientes base de datos: PubMed, ScienceDirect, SciELO, Springer, Google Scholar.

El periodo de búsqueda se seleccionó de acuerdo a un tiempo considerable para determinar la evolución de técnicas y medicamentos contra el $E$. faecalis. Los artículos seleccionados fueron a partir del año 2003 hasta diciembre de 2020.

La selección de artículos se formuló con base a los siguientes criterios de inclusión/exclusión. Criterios de inclusión: artículos en español y en inglés, artículos de acceso libre, artículos desde el año 2003 hasta el año 2020, artículos enfocados especialmente en E. faecalis. Criterios de exclusión: artículos sin base científica, artículos fuera del tema de estudio.

Después de realizar la búsqueda se seleccionaron 65 estudios. Finalmente se consideran 37 de ellos, con los que se realiza la revisión bibliográfica; excluyendo 28 estudios que no fueron relevantes para la investigación, por no guardar relación con los objetivos planteados.

Para extraer los datos, se utilizó un esquema de clasificación de los artículos, en una tabla de Excel que incluye la siguiente información (Tabla 1).

Tabla 1. Extracción de datos

\begin{tabular}{ll}
\hline Objetivo & Criterios de extracción \\
\hline $\begin{array}{ll}\text { Conocer la efectividad del gel de } \\
\text { Clorhexidina y pasta de Hidróxido de }\end{array}$ & Modo de acción \\
$\begin{array}{l}\text { Calcio con Clorhexidina como medica- } \\
\text { mento intraconducto en la eliminación }\end{array}$ & Tiempo de acción \\
del E. faecalis. & Efectividad antimicrobiana \\
\hline
\end{tabular}




\section{Revisión de la literatura}

\section{Enterococcus faecalis}

E. faecalis es una bacteria en forma de coco, Gram positiva, y su presencia en los túbulos dentinarios es la principal causa de infección en dientes con fracaso en el tratamiento endodóntico. Se caracteriza por ser resistente a medios poco nutritivos y a un $\mathrm{pH}$ alcalino ${ }^{6}$.

La presencia frecuente de E. faecalis en los canales radiculares en dientes con fracaso indica que se presenta un problema terapéutico significativo. Considerando los desafíos a los que se presenta el E. faecalis para salvaguardar su existencia, enfrentando diversos factores tales como: la capacidad de soportar la acción de agentes antimicrobianos y resistir a la falta de nutrientes en canales (limpios y obturados) en donde se ubica este microorganismo ${ }^{6}$.

\section{Medicación intraconducto}

La medicación Intraconducto (MI) se caracteriza por emplear un fármaco en los canales radiculares entre sesiones para concluir con éxito un tratamiento endodóntico, para eliminar la infección y proliferación de microorganismos dentro de los túbulos dentinarios, los que pueden conllevar al fracaso del tratamiento endodóntico. Las bacterias sobreviven en el canal radicular, por ello el uso de la MI complementa la asepsia, funcionando como una barrera física y química que impide la reinfección y aumenta el éxito del tratamiento. La MI más utilizada durante mucho tiempo ha sido el HC, seguido del formocresol, pastas medicadas de metronidazol, ciprofloxacina entre otros. Existen nuevas alternativas de MI a base de hierbas naturales como son el propóleo, gel de aloe vera, Morinda citrifolia (NONI) y el jengibre ${ }^{6-8}$.

Los objetivos de la Medicación Intracanal son los siguientes:

- Eliminar las bacterias que persisten en los canales radiculares.

- Contrarrestar los remanentes tóxicos que se encuentren en la pulpa.

- Reducir el proceso inflamatorio junto con el exudado periapical.

- Reducir la respuesta inflamatoria junto con el dolor ${ }^{8}$.

\section{Hidróxido de calcio}

El HC es considerado como el medicamento habitual utilizado para el tratamiento de canales infectados, siendo la primera medicación para el tratamiento pulpar Posee un efecto bactericida eficaz por el alto $\mathrm{pH}$ que inhibe el metabolismo bacteriano, crecimiento, división celular y se clasifica químicamente como una base fuerte, además tiene una actividad dentinogénica y osteogénica ${ }^{8,9}$.

El HC en la práctica endodóntica presenta una acción antiinflamatoria para prevenir la salida del exudado de los vasos sanguíneos hasta el ápice, así como una capacidad de desnaturalizar e hidrolizar las proteínas, con ello destruye el tejido blando remanente que se encuentra dentro de los canales, generando una buena asepsia y disminuyendo la filtración apical, lo que aportaría a concluir con un tratamiento endodóntico exitoso ${ }^{10}$.

\section{Clorhexidina}

El gluconato de clorhexidina fue utilizado por primera vez en 1954 en Gran Bretaña, la función fue como antiséptico para heridas de la piel y en 1959 se utilizó en la Odontología como desinfectante de la cavidad bucal; a partir del año 1970 se hizo comercial el uso de la CHX como enjuague bucal capaz de inhibir la formación de placa bacteriana y el desarrollo de gingivitis. Se reconoció a la CHX como un agente bucal antimicrobiano efectivo, el cual se usa como producto terapéutico en las infecciones de la boca. La CHX tiene un alto potencial cuando se utiliza como solución irrigadora o como medicamento intracanal durante el tratamiento endodóntico, teniendo ventajas antimicrobianas ${ }^{10,11}$.

Las principales propiedades de la aplicación en el tratamiento endodóntico son las siguientes: 1) Efecto bacteriostático y bactericida. 2) Actividad antimicrobiana de alto espectro contra bacterias Gram positivas y Gram negativas, 3) Sustantividad (capacidad antimicrobiana a largo plazo) ${ }^{11}$.

El mecanismo de acción se produce cuando la CHX es absorbida, generando así que esta llegue dentro de la pared celular de los microorganismos llegando a producir una filtración de los componentes intracelulares, de igual manera afecta a la barrera de permeabilidad de la pared celular. La CHX se absorbe gradualmente y es liberada durante 24 horas, por ello es que reduce la formación bacteriana de la superficie dental ${ }^{9}$.

\section{Eficacia del gel de clorhexidina e hidróxido de calcio}

Gómez et al. ${ }^{10}$, en el año 2003 realizó un estudio en el cual evaluaron la eficacia del gel de gluconato de $\mathrm{CHX}$ al $2 \%$ e $\mathrm{HC}$ como medicamento intracanal frente a $E$. faecalis. La muestra se realizó en tubos de dentina de incisivos bovinos infectados con E. faecalis. Teniendo como resultado que la $\mathrm{CHX}$ al 2\% inhibió completamente el crecimiento de E. faecalis en un tiempo promedio de 7 días. Mientras que Fernández et al. ${ }^{11}$ en el año 2018 evaluaron la susceptibilidad de las biopelículas de los microorganismos (E. faecalis. y Candida. albicans) en dientes bovinos. Se aplicó HC y CHX al 2\% como medicamentos intracanales. Los resultados obtenidos determinan que ninguno de los dos medicamentos resultó ser efectivos ante la eliminación del E. faecalis (Tabla 2).

Marickar et al. ${ }^{12}$ en el ańo 2014 realizaron un estudio in vitro para evaluar la efectividad antibacteriana del $\mathrm{HC}$ y la CHX al 2\% como medicamentos intracanales en la eliminación del E. faecalis. Las muestras fueron realizadas en dientes humanos unirradiculares extraídos e infectados. Este estudio menciona que realizó cultivo de este microorganismo en agar nutritivo durante un periodo de tiempo de 3, 5 y 7 días, teniendo como resultado que la 
CHX al 2\% fue el único medicamento intracanal que logro eliminar esta bacteria. Mientras que Mozayeni et al. ${ }^{13}$ en el mismo año evaluó la efectividad del HC y la CHX al 2\%. Teniendo como resultado que el gel de CHX al 2\% tuvo la máxima eficacia como medicamento intracanal contra el E. faecalis (Tabla 3).

Souza-Filho et al. ${ }^{14}$ en el año 2008 evaluó la efectividad de la $\mathrm{CHX}$ al 2\%, HC como medicamentos intracanales contra $E$. faecalis. La actividad antimicrobiana fue determinada mediante el método de difusión en agar. El $\mathrm{pH}$ de las pastas fue medido inmediatamente después de la preparación, luego de 24 horas y 1 semana después. Los resultados del estudio manifiestan que la CHX al $2 \%$ y el HC presentan actividad antimicrobiana, pero el medicamento más efectivo contra el microorganismo probado fue la CHX al 2\% (Tabla 3).

Valera et al. ${ }^{15}$ en el año 2009 evaluó la acción del HC y $\mathrm{CHX}$ al $2 \%$ como medicamento intracanal contra $E$. faecalis. El estudio se realizó en incisivos de humanos recién extraídos contaminados con E. faecalis durante 21 días. La muestra se dividió en 2 grupos y se aplicó la medicación intracanal durante 7 días. Los resultados fueron los siguientes: Tanto la CHX al 2\% e HC fueron efectivos en la eliminación de E. faecalis, manteniendo los canales radiculares libres de microorganismos (Tabla 4)
Delgado et al. ${ }^{16}$ en el año 2010 evaluó la efectividad de la medicación intracanal antes mencionada (HC, gel CHX al 2\%) en incisivos de humanos recién extraídos, infectados con $E$. faecalis los cuales se dividieron en 2 grupos y un grupo control, teniendo como resultados que el uso de la CHX al 2\% presentó de manera significativa una actividad antibacteriana seguido del HC (Tabla 4).

Krithikadatta et al. ${ }^{17}$ en el ańo 2007, Vaghela et al. ${ }^{18}$ en el 2011 y Vasudeva et al. ${ }^{19}$ en el 2017, evaluaron la desinfección de los túbulos dentinarios usando gel de $\mathrm{CHX}$ al $2 \%$ e HC contra $E$. faecalis. Realizaron el estudio en dientes humanos extraídos en los cuales colocaron la medicación en profundidades de $200 \mu \mathrm{m}$ y $400 \mu \mathrm{m}$ en un periodo de 21 días. Los resultados obtenidos en estos estudios indican que el gel de CHX al $2 \%$ obtuvo los mejores resultados antimicrobianos contra el $E$. faecalis a una profundidad de $400 \mu \mathrm{m}$ (Tabla 4).

Ooi et al. ${ }^{20}$ en el año 2018 realizó un estudio en piezas dentales humanas extraídas y se procedió a infectar con E. faecalis durante un periodo de tiempo de 21 días, posterior a la infección se analizaron muestras aleatorias y se procedió a clasificar en grupos de acuerdo al medicamento y realizando el análisis a profundidades de 200 $\mu \mathrm{m}$ y $400 \mu \mathrm{m}$. Los resultados de este estudio determinaron que todas las muestras con ambos medicamentos

Tabla 2. Resultados de estudios realizados en dientes bovinos extraídos contra E. faecalis

\begin{tabular}{ccccc}
\hline Autor & Año & Medicamento & Efectividad & Tiempo de acción \\
\hline Gómez et al. & 2003 & CHX 2\% - HC & CHX 2\%: 99,05\% & 7 días \\
Fernández et al. & 2018 & CHX 2\% - HC & NM & 7 días \\
\hline
\end{tabular}

NM: no menciona

Tabla 3. Resultados de estudios realizados in vitro contra $E$. faecalis

\begin{tabular}{ccccc}
\hline Autor & Año & $\begin{array}{c}\text { Medicamento } \\
\text { Utilizado }\end{array}$ & Efectividad & Tiempo de acción \\
\hline Marickar $e$ al. & 2014 & CHX 2\% - HC & CHX 2\%: $100 \%$ & 3,5 y 7 días \\
Mozayeni $e t$ al. & 2014 & CHX 2\% - HC & CHX 2\%: NM & 7 días \\
Souza-Filho et al. & 2008 & CHX 2\% - HC & CHX 2\%: $100 \%$ & 24 horas \\
\hline
\end{tabular}

NM: no menciona

Tabla 4. Resultados de estudios realizados en dientes humanos extraídos contra E. faecalis

\begin{tabular}{|c|c|c|c|c|c|}
\hline Autor & Año & $\begin{array}{c}\text { Medicamento } \\
\text { Utilizado }\end{array}$ & Efectividad & Profundidad & Tiempo de acción \\
\hline Valera et al. & 2009 & $\mathrm{CHX} 2 \%-\mathrm{HC}$ & $\mathrm{CHX} 2 \%-\mathrm{HC}$ & $\mathrm{NM}$ & 21 días \\
\hline Delgado et al. & 2010 & $\mathrm{CHX} 2 \%-\mathrm{HC}$ & $\mathrm{CHX} 2 \%-\mathrm{HC}$ & NM & NM \\
\hline Krithikadatta et al. & 2007 & $\mathrm{CHX} 2 \%-\mathrm{HC}$ & CHX $2 \%: 100 \%$ & $200 \mu \mathrm{m}-400 \mu \mathrm{m}$ & NM \\
\hline Vaghela et al. & 2011 & $\mathrm{CHX} 2 \%-\mathrm{HC}$ & $\mathrm{CHX} 2 \%$ & $200 \mu \mathrm{m}-400 \mu \mathrm{m}$ & 21 días \\
\hline Vasudeva et al. & 2017 & $\mathrm{CHX} 2 \%-\mathrm{HC}$ & $\mathrm{CHX} 2 \%$ & $200 \mu \mathrm{m}-400 \mu \mathrm{m}$ & 21 días \\
\hline Ooi et al. & 2018 & $\mathrm{CHX} 2 \%-\mathrm{HC}$ & $\mathrm{CHX} 2 \%-\mathrm{HC}$ & $200 \mu \mathrm{m}-400 \mu \mathrm{m}$ & 21 días \\
\hline Lakhani et al. & 2017 & $\mathrm{CHX} 2 \%-\mathrm{HC}$ & $\mathrm{CHX} 2 \%$ & NM & 2 y 7 días \\
\hline Valverde et al. & 2017 & $\mathrm{CHX} 2 \%-\mathrm{HC}$ & CHX $2 \%: 100 \%$ & NM & 24 horas \\
\hline Yadav et al. & 2018 & $\mathrm{CHX} 2 \%-\mathrm{HC}$ & $\mathrm{CHX} 2 \%$ & NM & 21 días \\
\hline
\end{tabular}

NM: no menciona 
tuvieron el mismo efecto antimicrobiano contra el $E$. faecalis (Tabla 4).

Lakhani et al. ${ }^{21}$ y Valverde et al. ${ }^{22}$ en el 2017 realizaron una comparación de la actividad antimicrobiana de diferentes medicamentos intraconductos los cuales fueron: Pasta triple antibiótica (ATP), HC, y CHX al 2\%. Las muestras fueron infectadas con $E$. faecalis durante un periodo de 3 semanas. Para el análisis de estos estudios se clasificaron las muestras en grupos de acuerdo a la medicación intracanal utilizada en cada caso durante un periodo de 2-7 días. Obteniendo como resultado que el gel de $\mathrm{CHX}$ al $2 \%$ resultó ser el medicamento intracanal con mayor actividad y eficacia contra el E. faecalis (Tabla 4).

Yadav et al. ${ }^{23}$ en el 2018, comparó la efectividad antimicrobiana del $\mathrm{HC}$, $\mathrm{CHX}$ al $2 \%$ y una formulación a base de curcumina contra el E. faecalis. El estudio se realizó en dientes extruidos, previo a la contaminación de $E$. faecalis, se realizó una desinfección en autoclave. Terminado el proceso se procedió a la contaminación y se incubó durante 24 horas a $37^{\circ} \mathrm{C}$. En los resultados de este estudio se determinó que la CHX al $2 \%$ obtuvo el mejor efecto antibacteriano, seguido de la curcumina, mientras que el HC tuvo un efecto negativo para la eliminación del E. faecalis (Tabla 4).

Barbosa et al. ${ }^{24}$ en el año 2018 realizó una investigación in vivo de los efectos de la medicación intracanal a base de HC sobre los niveles de bacterias, la muestra se realizó en dientes unirradiculares. Los 20 canales infectados se dividieron en dos grupos; Grupo 1: CHX al 2\% y Grupo 2: Hipoclorito de Sodio (NaCIO) al 6\%. Se utilizó una medicación intracanal a base de $\mathrm{HC}$ recién preparada durante un periodo de 30 días. Teniendo como resultado que la $\mathrm{CHX}$ al 2\%, el $\mathrm{NaCIO}$ al $6 \%$ y el $\mathrm{HC}$ tuvieron un efecto positivo en la reducción bacteriana como medicación intracanal (Tabla 5).

\section{Eficacia de gel de clorhexidina en combinación con hidróxido de calcio}

Evans et al. ${ }^{25}$ en el año 2003, Siren et al. ${ }^{26}$ en el 2004 y Dohyun et al. ${ }^{27}$ en el 2015 evaluaron la actividad antimicrobiana en incisivos bovinos infectados con E. faecalis. Evans et al. ${ }^{25}$ aplico en su estudio HC combinado con gel de CHX al 2\% como medicamento intracanal en un periodo de 7 días en donde el E. faecalis no tuvo crecimiento bacteriano al aplicar la mezcla de medicamentos. Mientras que en los estudios de Siren et al. ${ }^{26}$ y Dohyun et al. ${ }^{27}$ a los 14 días el HC por si solo obtuvo una eficacia del $70 \%$ en la eliminación del E. faecalis con una probabilidad de que disminuya su efectividad antimicrobiana con el tiempo, en comparación a la mezcla de $\mathrm{HC}$ y gel de $\mathrm{CHX}$ al $2 \%$ que obtuvo una efectividad del $90 \%$. Teniendo como resultado que la combinación de $\mathrm{HC}$ junto con el gel de CHX al 2\% fue significativamente eficaz para eliminar $E$. faecalis de los túbulos dentinarios en los estudios antes mencionados (Tabla 6).

Figueiredo et al. ${ }^{28}$ en el año 2006, Ghabraei et al. ${ }^{29}$ en el 2018 y Punathil et al. ${ }^{30}$ en el 2020 evaluaron la actividad antimicrobiana del $\mathrm{HC}$ combinado con $\mathrm{CHX}$ al $2 \%$. Se aplicó los medicamentos en las muestras infectados con E. faecalis. En el estudio de Figueiredo et al. ${ }^{28}$ utilizaron dos métodos: la prueba de difusión en agar y la prueba de contacto directo. El HC + CHX al 2\% requirió de 30 segundos a 6 horas para eliminar todos los microorganismos analizados, mientras que en los estudios de Ghabraei et al. ${ }^{29}$ y Punathil et al. ${ }^{30}$ no indican el tiempo de efectividad. En el estudio de Figueiredo et al. ${ }^{28}$ la pasta de $\mathrm{HC}+\mathrm{CHX}$ al $2 \%$ al tercer día pudo eliminar completamente el E. faecalis. Los resultados obtenidos para los diferentes estudios coincidieron que la mezcla de hidróxido de calcio y CHX al 2\% fue la más eficaz en todas sus concentraciones (Tabla 7).

Tabla 5. Resultados de estudios realizados in vivo contra E. faecalis

\begin{tabular}{ccccc}
\hline Autor & Año & $\begin{array}{c}\text { Medicamento } \\
\text { Utilizado }\end{array}$ & Efectividad & Tiempo de acción \\
\hline Barbosa $e t$ al. & 2018 & CHX $\%$ - HC & CHX $2 \%-$ HC: $99,03 \%$ & 30 días \\
\hline
\end{tabular}

Tabla 6. Resultados de estudios realizados en dientes bovinos extraídos contra E. faecalis

\begin{tabular}{ccccc}
\hline Autor & Año & $\begin{array}{c}\text { Medicamento } \\
\text { utilizado }\end{array}$ & Efectividad & Tiempo de acción \\
\hline Evans et al. & 2003 & CHX 2\% combinado con HC & CHX al 2\% + HC: NM & 7 días \\
Siren et al. & 2004 & CHX 2\% combinado con HC & CHX 2\% + HC: $90 \%$ & 14 días \\
Dohyun $e t$ al. & 2015 & CHX 2\% combinado con HC & CHX 2\% + HC: $90 \%$ & 14 días \\
\hline
\end{tabular}

NM: no menciona

Tabla 7. Resultados de estudios realizados en dientes humanos extraídos contra E. faecalis

\begin{tabular}{ccccc}
\hline Autor & Año & $\begin{array}{c}\text { Medicamento } \\
\text { Utilizado }\end{array}$ & Efectividad & Tiempo de acción \\
\hline Figueiredo et al. & 2006 & CHX 2\% combinado con HC & CHX 2\%+HC: $100 \%$ & 6 horas \\
Ghabraei et al. & 2018 & CHX 2\% combinado con HC & CHX 2\% + HC: NM & NM \\
Punathil et al. & 2020 & CHX 2\% combinado con HC & CHX 2\% + HC: NM & NM \\
Samiei $e$ al. & 2018 & CHX 0,12 combinado con HC & CHX 0,12\% + HC: 89,65\% & 3,7 y 14 días \\
\hline
\end{tabular}

NM: no menciona 
Samiei et al. ${ }^{10}$ en el ańo 2018 comparó los efectos antibacterianos de un gel que contienen óxido de zinc y una mezcla de $\mathrm{HC}$ y $\mathrm{CHX}$ al $0,12 \%$ como medicación intracanal, las muestra se infectaron con $E$. faecalis. Utilizaron los medicamentos intracanales en periodos de 3 , 7 y 14 días, las muestras fueron divididas en 4 grupos, después del tiempo especificado se retiró la medicación intracanal para proceder al recuento final de bacterias. Los datos se analizaron entre los grupos de muestras en los diferentes intervalos de tiempo. Los resultados indicaron que la mezcla de $\mathrm{HC}$ con $\mathrm{CHX}$ como medicamento intracanal fue más eficaz contra el $E$. faecalis (Tabla 7).

\section{Conclusiones}

En base a los resultados obtenidos de los diferentes estudios a lo largo de los años sobre la medicación intracanal a base de gel de clorhexidina e hidróxido de calcio se concluye que: la clorhexidina y el hidróxido de calcio tienen un tiempo promedio de acción de alrededor 7 días para la eliminación total del E. faecalis.

La clorhexidina al 2\% resultó ser un medicamento intracanal más utilizado y con resultados favorables, siendo efectiva como medicación intraconducto en la eliminación de microorganismos entre ellos del E. faecalis. Mientras que el hidróxido de calcio a pesar de tener excelentes propiedades antimicrobianas no resulto capaz de eliminar el $E$. faecalis en su totalidad, por lo tanto, en la presente revisión no se recomienda su uso como medicación intraconducto. Por último, la combinación de hidróxido de calcio más clorhexidina demostró, en un menor porcentaje de estudios, ser un medicamento intracanal efectivo en la eliminación del E. faecalis.

\section{Referencias bibliográficas}

1. C, Oporto G. Implicancias clínicas de la contaminación microbiana por Enterococcus faecalis en canales radiculares de dientes desvitalizados: Revisión de la literatura. Rev Odontológica Mex. 2015;19(3):181-6. DOI:10.1016/j. rodmex.2015.04.002.

2. Pardi G, Guilarte C, Cardozo E. ENB. Detección de Enterococcus faecalis en dientes con fracaso en el tratamiento endodóntico. Rev Acta Odontológica Venezolana. 2009;47(1):1-11.

3. Mohammadi Z, Dummer PMH. Properties and applications of calcium hydroxide in endodontics and dental traumatology. Int Endod J. 2011;44(8):697-730. DOI: $10.1111 /$ j.1365-2591.2011.01886.x.

4. Saatchi M, Shokraneh A, Navaei H, Maracy MR, Shojaei H. Antibacterial effect of calcium hydroxide combined with chlorhexidine on Enterococcus faecalis: A systematic review and meta-analysis. J Appl Oral Sci. 2014;22(5):356-65. DOI: 10.1590/1678775720140032

5. Bonza MPV, Chacón VM, Pérez BED, Sandoval LVH, Plata MEV, Angarita AB, et al. Evaluación in vitro de la eficacia del digluconato de clorhexidina en gel al 0,2\% y $2 \%$ como medicación intraconducto frente a Candida albicans. UstaSalud 2013;12(2):83-90.DOI:10.15332/ us.v12i2.1211
6. Pedraza M. Medicación intraconducto frente al Enterococcus faecalis. Rev Odontológica Basadrina. 2020;3(2):49_ 55. DOI:10.33326/26644649.2019.3.2.893

7. Chavez M. Medicación intraconducto empleada con más frecuencia en la terapia endodóntica: revisión bibliográfica. [tesis de bachiller]. [Ecuador]; Facultad de Odontología, Universidad de las Américas; 2019. 62 p.

8. Heredia Bonetti JM, Sosa SAR. Uso de la Clorhexidina en Endodoncia: revisión de la literatura. Rev Asoc Odontol Argent. 2005;93(3):245-248.

9. Burgos F. Medicación intraconducto en endodoncia. [tesis doctoral]. [Chile]; Facultad de Odontología, Universidad Valparaiso. 2013. 37 p.

10. Samiei M, Torab A, Hosseini O, Abbasi T, Abdollahi AA, Divband B. Antibacterial effect of two nano zinc oxide gel preparations compared to calcium hydroxide and chlorhexidine mixture. Iran Endod J. 2018;13(3):30511. DOI: $10.22037 /$ iej.v13i3.19866

11. Gomes B, Souza S, Ferraz C, Teixeira F, Zaia A, Valdrighi L. Effectiveness of $2 \%$ chlorhexidine gel and calcium hydroxide against Enterococcus faecalis in bovine root dentine in vitro. Int Endod J. 2003;36(4):267-75. DOI: 10.1046/j.1365-2591.2003.00634.x

12. Zancan RF, Calefi PHS, Borges MMB, Lopes MRM, de Andrade FB, Vivan RR, et al. Antimicrobial activity of intracanal medications against both Enterococcus faecalis and Candida albicans biofilm. Microsc Res Tech. 2018;82(5):494-500. DOI: 10.1002/jemt.23192.

13. Marickar RF, Geetha RV, Neelakantan P. Efficacy of contemporary and novel intracanal medicaments against Enterococcus faecalis. J Clin Pediatr Dent. 2014;39(1):4750. DOI: $10.17796 /$ jcpd.39.1.wmw9768314h56666.

14. Mozayeni MA, Haeri A, Dianat O, Jafari AR. Antimicrobial effects of four intracanal medicaments on Enterococcus faecalis: An in vitro study. Iran Endod J. 2014;9(3):195-8.

15. De Souza-Filho FJ, Soares A, Vianna ME, Zaia AA, Ferraz CCR, Gomes BPF. Antimicrobial effect and $\mathrm{pH}$ of chlorhexidine gel and calcium hydroxide alone and associated with other materials. Braz Dent J. 2008;19(1):2833. DOI: $10.1590 /$ S0103-64402008000100005.

16. Valera MC, da Silva KCG, Maekawa LE, Carvalho CAT, Koga-Ito CY, Camargo CHR, et al. Antimicrobial activity of sodium hypochlorite associated with intracanal medication for Candida albicans and Enterococcus faecalis inoculated in root canals. J Appl Oral Sci. 2009;17(6):5559. DOI: $10.1590 / \mathrm{s} 1678-77572009000600003$.

17. Delgado RJR, Gasparoto TH, Sipert CR, Pinheiro CR, Moraes IG, Garcia RB, et al. Antimicrobial Effects of Calcium Hydroxide and Chlorhexidine on Enterococcus faecalis. J Endod. 2010;36(8):1389-93. DOI: 10.1016/j. joen.2010.04.013.

18. Krithikadatta J, Indira R, Dorothykalyani AL. Disinfection of Dentinal Tubules with 2\% Chlorhexidine, 2\% Metronidazole, Bioactive Glass when Compared with Calcium Hydroxide as Intracanal Medicaments. J Endod. 2007;33(12):1473-6. DOI: 10.1016/j. joen.2007.08.016. 
19. Vaghela DJ, Kandaswamy D, Venkateshbabu N, Jamini N, Arathi G. Disinfection of dentinal tubules with two different formulations of calcium hydroxide as compared to 2\% chlorhexidine: As intracanal medicaments against Enterococcus faecalis and Candida albicans: An in vitro study. J Conserv Dent. 2011;14(2):182-6. DOI: 10.4103/0972-0707.82625.

20. Vasudeva A, Sinha DJ, Tyagi SP, Singh NN, Garg P, Upadhyay D. Disinfection of dentinal tubules with 2\% Chlorhexidine gel, Calcium hydroxide and herbal intracanal medicaments against Enterococcus faecalis: An in-vitro study. Singapore Dent J. 2017; 38:39-44. DOI: 10.1016/j.sdj.2017.06.001.

21. Lakhani AA, Sekhar KS, Gupta P, Tejolatha B, Gupta A, Kashyap S, et al. Efficacy of Triple Antibiotic Paste, Moxifloxacin, Calcium Hydroxide And 2\% Chlorhexidine Gel in Elimination of Enterococcus faecalis: An In vitro Study. J Clin Diagn Res. 2017;11(1): ZC06-9. DOI: 10.7860/JCDR/2017/22394.9132.

22. Valverde ME, Baca P, Ceballos L, Fuentes MV, Ruiz-Linares M, Ferrer-Luque CM. Antibacterial efficacy of several intracanal medicaments for endodontic therapy. Dent Mater J. 2017;36(3):319-24. DOI: 10.4012/ dmj.2016-102.

23. Yadav R, Tikku A, Chandra A, Verma P, Bains R, Bhoot $\mathrm{H}$. A comparative evaluation of the antimicrobial efficacy of calcium hydroxide, chlorhexidine gel, and a curcumin-based formulation against Enterococcus faecalis. Natl J Maxillofac Surg. 2018,9(1):52. DOI: 10.4103/njms. NJMS_47_17.

24. Barbosa M, Arruda-Vasconcelos R, De-Jesus-Soares A, Zaia AA, Ferraz CCR, de Almeida JFA, et al. Effectiveness of calcium hydroxide-based intracanal medication on infectious/inflammatory contents in teeth with post-treatment apical periodontitis. Clin Oral Investig. 2018;23(6):2759-66. DOI: 10.1007/s00784-0182719-0.
25. Evans MD, Baumgartner JC, Khemaleelakul SU, Xia T. Efficacy of calcium hydroxide: Chlorhexidine paste as an intracanal medication in bovine dentin. J Endod. 2003;29(5):338-9. DOI: 10.1097/00004770200305000-00005.

26. Sirén EK, Haapasalo MPP, Waltimo TMT, Ørstavik D. In vitro antibacterial effect of calcium hydroxide combined with chlorhexidine or iodine potassium iodide on Enterococcus faecalis. Eur J Oral Sci. 2004;112(4):32631. DOI: $10.1111 /$ j.1600-0722.2004.00144.x.

27. Kim D, Kim E. Antimicrobial effect of calcium hydroxide as an intracanal medicament in root canal treatment: a literature review - Part II. in vivo studies. Restor Dent Endod. 2015;40(2):97. DOI: 10.5395/rde.2015.40.2.97.

28. Figueiredo A, Vianna ME, Sena NT, Zaia AA, Ferraz CCR, de Souza Filho FJ. In vitro evaluation of the antimicrobial activity of calcium hydroxide combined with chlorhexidine gel used as intracanal medicament. Oral Surgery Oral Med Oral Pathol Oral Radiol Endod. 2006;102(4):544-50. DOI: 10.1016/j. tripleo.2006.04.010.

29. Ghabraei S, Bolhari B, Sabbagh MM, Afshar MS. Comparison of Antimicrobial Effects of Triple Antibiotic Paste and Calcium Hydroxide Mixed with 2\% Chlorhexidine as Intracanal Medicaments Against Enterococcus faecalis Biofilm. J Dent (Tehran). 2018;15(3):151-160.

30. Punathil S, Moyin S, Bhat SS, Hedge S, Pai A, James J. Comparison of antibacterial effect of calcium hydroxide combined with chlorhexidine and povidone-iodine against Enterococcus faecalis in dentinal tubules of human incisors: An in vitro comparative study. J Pharm Bioallied Sci. 2020;12(5):S448-52. DOI: 10.4103/jpbs. JPBS_134_20. 